\title{
Empfehlungen zur Durchführung einer Bronchoskopie in Zeiten der COVID-19-Pandemie
}

\section{Recommendations for Performing Bronchoscopy in Times of the COVID-19 Pandemic}

Autoren

K. Darwiche1, B. Ross², W. Gesierich ${ }^{3}$, C. Petermann ${ }^{4}$, R.-H. Huebner ${ }^{5}$, C. Grah ${ }^{6}$, D. Gompelmann? , J. Hetzel ${ }^{8}$, A. Holland ${ }^{9}$, S. Eisenmann ${ }^{10}$, F. Stanzel ${ }^{11}$, A. Kempa ${ }^{12}$, K. Conrad ${ }^{13}$, R. Eberhardt ${ }^{14,15}$

Institute

1 Klinik für Pneumologie, Sektion Interventionelle Bronchologie, Ruhrlandklinik - Universitätsmedizin Essen

2 Krankenhaushygiene, Universitätsmedizin Essen

3 Lungenzentrum am Helios Klinikum München West

4 Klinik für Pneumologie, Asklepios Klinikum Harburg, Hamburg

5 Medizinische Klinik mit Schwerpunkt Infektiologie und Pneumologie, Charité - Universitätsmedizin Berlin

6 Abteilung für Pneumologie, Gemeinschaftskrankenhaus Havelhöhe, Berlin

7 Klinische Abteilung für Pulmologie, Universitätsklinik für Innere Medizin II, Medizinische Universität Wien, Österreich

8 Klinik für medizinische Onkologie und Pneumologie, Universitätsklinikum Tübingen

9 Klinik für Innere Medizin SP Pneumologie, Universitätsklinikum Gießen und Marburg

10 Klinik für Innere Medizin I, Universitätsklinikum Halle (Saale)

11 Pneumologie - Thorakale Endoskopie, Lungenklinik Hemer

12 Pneumologie, Medizinische Klinik I, Klinik Löwenstein

13 Pneumologie, Lungenklinik Ballenstedt/Harz

14 Pneumologie und Beatmungsmedizin, Thoraxklinik am Universitätsklinikum Heidelberg

15 Translational Lung Research Center Heidelberg (TLRCH), Deutsches Zentrum für Lungenforschung (DZL)

Bibliografie

DOI https://doi.org/10.1055/a-1154-1814 |

Online-Publikation: 14.4.2020 |

Pneumologie 2020; 74: 260-262

(c) Georg Thieme Verlag KG Stuttgart · New York

ISSN 0934-8387
Korrespondenzadresse

PD Dr. med. Kaid Darwiche, Deutsche Gesellschaft für Pneumologie, Sprecher der Sektion 2 „Endoskopie“, Pneumologische Universitätsklinik, Ruhrlandklinik Universitätsmedizin Essen, Tüschener Weg 40, 45239 Essen kaid.darwiche@rlk.uk-essen.de

\section{ZUSAMMENFASSUNG}

COVID-19, ausgelöst durch Coronavirus SARS-CoV-2, ist eine neue, sich pandemisch ausbreitenden Infektionserkrankung, welche aktuell weltweit zu einer erheblichen Belastung der Gesundheitssysteme geführt hat. Krankenhausmitarbeiter stellen in Ländern mit hoher Krankheitsaktivität, wie China oder Italien, einen signifikanten Anteil der an COVID-19 Erkrankten und Verstorbenen dar. Bronchoskopische Untersuchungen führen beim untersuchten Patienten zu Hustenreiz und Aerosolbildung und beinhalten daher ein erhöhtes Infektionsrisiko für das Untersuchungsteam und für andere Patienten. In der aktuellen Pandemie sind daher besondere Maßnahmen bei der Durchführung bronchoskopischer Untersuchungen zu beachten, um das Infektionsrisiko zu senken.

\section{ABSTRACT}

COVID-19, caused by coronavirus SARS-CoV-2 is a new and ongoing infectious disease affecting healthcare systems worldwide. Healthcare worker are at high risk for COIVD19 and many have been infected or even died in countries severely affected by COVID-19 like China or Italy. Bronchoscopy causes cough and aerosol production and has to be considered a significant risk for the staff to get infected. Particular recommendations should guide to prevent spreading COVID-19 and to protect healthcare worker when performing a bronchoscopy. 
Das neuartige Coronavirus SARS-CoV-2 führt seit Anfang Dezember 2019, beginnend in der chinesischen Stadt Wuhan, zu einer bisher steigenden Anzahl an Infektionen weltweit, genannt COVID-19. Betroffene Patienten klagen am Häufigsten über Fieber und Husten, bei schweren Verläufen auch über Dyspnoe. Gastrointestinale Symptome können selten zusätzlich auftreten [1]. $80 \%$ der COVID-19-Infektionen verlaufen mild, intensivpflichtige Verläufe treten in $5 \%$ auf [2]. Im Gesundheitswesen tätige Personen machen bis zu $1 / 3$ der an COVID-19 erkrankten Patienten aus [3].

Der wesentliche Übertragungsweg von SARS-CoV-2 erfolgt als Tröpfcheninfektion, sodass COVID-19-Erkrankte oder -Verdächtige neben Schutzkitteln, Handschuhen und Schutzbrillen nur mit FFP-Masken betreut werden sollten. Bei rasch ansteigenden Infektionszahlen können die Infektionsketten nicht mehr sicher identifiziert und unterbrochen werden, sodass insbesondere milde Verlaufsformen, die $80 \%$ der Fälle ausmachen, nicht immer sicher identifiziert werden können. Auf der anderen Seite gehört die Bronchoskopie zu den Prozeduren, die im besonderen Maße zu einer ausgeprägten Aerosolbildung führen und somit ein hohes Infektionsrisiko bedeuten. Nach Vorgaben des „Centers for Disease Control and Prevention“ sollte im Falle eines Mangels an Schutzmasken diese prioritär für Prozeduren mit ausgeprägter Aerosolbildung wie Bronchoskopien eingesetzt werden [4].

Diese Vorgaben dienen dazu, bronchoskopisch tätigen Ärzten Handlungsempfehlungen an die Hand zu geben, um in der aktuellen COVID-19-Pandemie betroffene und nicht betroffene Patienten zu schützen, das Risiko von Übertragungen zu minimieren sowie die Aufrechterhaltung der klinischen Versorgung aller Patienten und die Verfügbarkeit von persönlichen Schutzausrüstungen sicherzustellen.

\section{Generelle Empfehlungen zu Schutz- maßnahmen bei allen Bronchoskopien}

- Strenge Indikationsstellung zur Durchführung von Bronchoskopien; Elektiveingriffe sollten verschoben werden

- Reduktion der Mitarbeiter (Bronchoskopiker, Bronchoskopieassistenz, ggf. Anästhesieteam) auf ein Kernteam, die alle Eingriffe des Tages durchführen

- Keine Besucher, Studierende, Aus- oder Weiterzubildende im Untersuchungsraum

- Nutzung von Einmalschutzkittel, Einmalhandschuhen und Mund-Nasen-Schutzmaske aller Anwesenden im Untersuchungsraum

- In Regionen mit hoher COVID-19-Prävalenz Nutzung einer personenbezogenen FFP2-Maske auch bei der Bronchoskopie nicht-verdächtiger Patienten durchgehend über mehrere Untersuchungen desselben Tages (Wechsel nur, wenn die Maske beschädigt, beschmutzt oder feucht geworden ist); dies gilt für alle an der Bronchoskopie unmittelbar Beteiligten

- Berücksichtigung der RKI-Richtlinie zum An- und Ablegen der Schutzmaske [5]

- Nutzung einer personenbezogenen Einmalschutzbrille (nach Aufbereitung auch an Folgetagen verwendbar) oder eines wiederverwendbaren Schutzvisiers (je nach Verfügbarkeit) bei Arzt und Bronchoskopieassistenz

\section{Vermeidung und Reduktion von Aerosolbildung}

- Starre Bronchoskopie mit JET-Ventilation, soweit medizinisch vertretbar, durch geschlossene Beatmungssysteme (Tubus, Larynxmaske) ersetzen

- Falls starre Bronchoskopie unvermeidbar, dann Nutzung mit konventioneller Beatmung und Reduktion des Austritts von Aerosol, z. B. mittels FLUVOG-Aufsatz

- Bei flexiblen Bronchoskopien transnasalen Zugang bevorzugen und zusätzliche Nutzung eines geschlitzten MundNasen-Schutzes für den Patienten

\section{Umgang bei gesichertem Nachweis von SARS-CoV-2 (COVID-19)}

- Die Entnahme von Proben der oberen Atemwege über Nasopharyngealabstriche ist die primäre und bevorzugte Diagnosemethode.

- Da es sich um ein Aerosol erzeugendes Verfahren handelt, das ein erhebliches Risiko darstellt, sollte die Indikation zur Bronchoskopie bei der Diagnose von COVID-19 streng gestellt und nur in Betracht gezogen werden, wenn

1. der Nasopharyngealabstrich negativ ist und klinisch eine hohe Wahrscheinlichkeit für COVID-19 besteht,

2. andere Diagnosen in Betracht gezogen werden, die das klinische Management erheblich verändern würden oder 3. eine dringende lebensrettende Intervention notwendig ist (Haemoptoe, höhergradige benigne oder maligne zentrale Atemwegsstenose oder Fremdkörperaspiration).

- Bei intubierten Patienten reicht die Entnahme von Trachealsekret. Im Falle der Bronchoskopie reicht die Entnahme von Bronchialsekret oder eine Bronchiallavage mit 2-3 ml NaCl. Eine BAL ist nicht notwendig und wird zur Diagnostik nicht empfohlen [7].

\section{Durchführung der Bronchoskopie}

- Unter Abwägung der Erfahrung von Untersucher und Bronchoskopieassistenz und dem klinischen Zustand des Patienten kann, von der S3-Leitlinie abweichend, die Untersuchung mit 2 Personen im Raum durchgeführt werden, wenn sichergestellt ist, dass eine dritte, erfahrene Person in Rufweite ist.

- Die Bronchoskopie sollte außerhalb von Notfällen immer innerhalb der Regelarbeitszeit und durch erfahrenes Personal (Untersucher/Assistenz) durchgeführt werden.

- Die Untersuchung sollte, außer bei intensivpflichtigen Patienten, in den Räumen der Bronchologie (Saal 1 oder 2; technische Raumluftanlage vorhanden) durchgeführt werden.

- Alle Mitarbeiter im Saal tragen als persönliche Schutzausrüstung Handschuhe, Einmalkittel, Haube, FFP3-Maske und eine Schutzbrille (Visier falls verfügbar). 
- Auf die Maßnahmen zum ordnungsgemäßen An- und Ausziehen der persönlichen Schutzausrüstung (laut RKI und lokalen Guidelines) ist zu achten.

- Bei zeitnaher und validierter Aufbereitung der Bronchoskopie, besteht kein Anhalt, dass der Aufbereitungsprozess zur Desinfektion der Geräte bei Coronaviren geändert werden muss [6].

\section{Interessenkonflikt}

Die Autorinnen/Autoren geben an, dass kein Interessenkonflikt besteht.

\section{Literatur}

[1] Guan W], Ni ZY, Hu Y et al.; China Medical Treatment Expert Group for Covid-19. Clinical Characteristics of Coronavirus Disease 2019 in China. N Engl J Med 2020. doi:10.1056/NEJMoa2002032

[2] Wu Z, McGoogan JM. Characteristics of and Important Lessons From the Coronavirus Disease 2019 (COVID-19) Outbreak in China: Summary of a Report of 72314 Cases From the Chinese Center for Disease Control and Prevention. JAMA 2020. doi:10.1001/jama.2020.2648

[3] Wang D, Hu B, Hu C et al. Clinical Characteristics of 138 Hospitalized Patients With 2019 Novel Coronavirus-Infected Pneumonia in Wuhan, China. JAMA 2020. doi:10.1001/jama.2020.1585

[4] https://www.cdc.gov/coronavirus/2019-ncov/infection-control/control-recommendations.html (aufgerufen am 17.03.2020)

[5] https://www.rki.de/DE/Content/InfAZ/N/Neuartiges_Coronavirus/ Ressourcen_schonen_Masken.pdf (aufgerufen am 17.03.2020)

[6] https://www.cdc.gov/infectioncontrol/guidelines/disinfection/healthcare-equipment.html (aufgerufen am 17.03.2020)

[7] Wahidi M, Lamb C, Murgu S et al. American Association for Bronchology and Interventional Pulmonology (AABIP). Statement on the Use of Bronchoscopy and Respiratory Specimen Collection in Patients with Suspected or Confirmed COVID-19 Infection). J Bronchology Interv Pulmol 2020. doi:10.1097/LBR.0000000000000681 